(Aus dem physiologischen Institut zu Königsberg i. Pr.)

\title{
Weitere Untersuchungen über das Verhalten der Froschlarven im galvanischen Strome.
}

Von

\section{Hermann.}

Die im vorigen Jahre beschriebene merkwiirdige Erscheinung, dass Froschlarven, besonders in einem gewissen Entwicklungsstadium, sich in einem galvanisch durchströmten Wasserbehälter mit dem Kopfe gegen die Anode einstellen ${ }^{1}$ ), habe ich auch in diesem Frubling wieder vielfach beobachtet, und den Versuch in mannigfacher Weise modificirt. Auch Herr Neubauer, welcher im Anschluss an die beschriebene Erscheinung eine grössere Versuchsreihe iiber das Verhalten von Thieren und Gliedmassen bei Massendurchströmung angestellt hat ${ }^{2}$ ), hat sich mehrfach mit dem Verhalten der Froschlarven beschäftigt, und einzelne der im Folgenden mitgetheilten Thatsachen ebenfalls, und unabhängig von mir, beobachtet.

Zunächst schien es wünschenswerth festzustellen, ob die galvanotropische Reaction vom Nervensystem, oder von einer rein musculären Einwirkung herrihrt. Ich versuchte daher das Nervensystem durch Vergiftung mit Curare auszuschalten. Allein die Larven (etwa 14 tägig) zeigten sich gegen Curare in dem Grade immun, dass sie fast 8 Tage in einer ziemlich starken Curarelösung lebend blieben und sich munter bewegten. Auch Morphium zeigte eine auffallend schwache Wirkung.

Es wurde nun versucht das Centralnervensystem operativ ganz oder theilweise auszuschalten.

1) Vgl. dies Archiv Bd. XXXVII. S. 457.

2) Diese noch nicht abgeschlossene Arbeit erstreckt sich in ihrom Haupttheil auf andere Fragen als die hier behandelte, und wird von Herrn $\mathrm{Neubauer}$ in seiner Dissertation mitgetheilt werden. 
Köpft man eine 8-10 Tage alte Larve vor den Kiemenbüscheln, so zeigt sie immer noch die galvanotropische Reaction, oder wenigstens die Unruhe und das Schlängeln so lange sie mit dem Kopfende nach der Cathode zu liegt, und sofortige Beruhigung, wenn man das Kopfende nach der Anode wendet (vgl. die frühere Mittheilung a. a. O. S. 458).

Schneidet man einer Larve den Schwanz dicht am Rumpfe ab, so zeigt der Rumpf kein Umlegen nach der Anode mehr; für diese Bewegung ist der Schwanz, das einzige Locomotionsorgan der Larve, unentbehrlich. Aber der Rumpf zeigt bei homodromer Lage (so sei der Kïrze halber diejenige Lage bezeichnet, bei welcher das Kopfende dem Strome nach gewendet ist, $d$. h. der Cathode zu liegt) lebhafte Unruhe, welche sich namentlicb in Hin- und Herpendeln des Schwanzstiimpfehens zu erkennen giebt. Sowie man den Rumpf in antidrome Lage bringt (Kopfende nach der Anode), so tritt vollkommene Ruhe ein.

Ebenso aber zeigt der abgeschnittene Schwanz in homodromer Lage regelmässig ein anhaltendes Schlängeln, und beruhigt sich in antidromer Lage augenblicklich.

Schneidet man dagegen den Schwanz etwa in der Mitte seiner Länge $a b$, so zeigt das hintere Ende keinerlei Einwirkung des Stromes mehr, während der Rumpf mit dem vorderen Schwanztheil sich antidrom einstellt, und der vordere Schwanztheil, für sich isolirt, bei homodromer Lage deutliches Schlängeln darbietet.

Das abgeschnittene hintere Schwanzende ist noch reich an Muskeln, welche longitudinal zwischen den sagittalen Segmentknorpeln ausgespannt sind; es enthält dagegen kein Rückenmark, wäbrend der vordere Schwanztheil noch einen caudalen Theil des Rückenmarks enthält. Der Versuch beweist also, dass die galvanotropische Reaction nicht musculärer, sondern centraler Natur, und an die Existenz des Rückenmarks gebunden ist; das Gehirn ist, wie die Versuche zeigen, für ihr Zustandekommen nicht nöthig, namentlich in der rudimentären Form des Schlängelns bei homodromer, und der Ruhe bei antidromer Lage.

Die centrale Natur der Erscheinung zeigt sich auch schon durch ihren ganzen Habitus. Bei Schliessung eines starken Stromes gerathen alle im Troge enthaltenen Larven in ein äusserst lebhaftes Hin- und Herschiessen, wie schon früher angegeben wurde, und beruhigen sich erst nachdem sie die antidrome Lage gefun- 
den haben. Bei diesem Hin- und Herschiessen sieht man oft eine einzelne Larve sich gewaltsam abwechselnd nach rechts und nach links krümmen, so dass Kopf und Schwanz sich fast beriihren, beinahe als $o b$ sie sich im Schmerze wände. Es lässt sich gewiss kein Modus denken, wie irgendwelche polare Reaction der Musculatur solche Bewegungen hervorrufen könnte.

In einer anderen Versuchsreihe legte ich nur einen Electrodendraht dauernd an die eine Schmalseite des Troges und tauchte den anderen bei sonst geschlossenem Kreise piötzlich in der Nähe einer Larve in das Wasser ein. Hierbei zeigt sich folgendes:

Ist die bewegliche Electrode die Cathode, und wird sie plötzlich unmittelbar am Schwanzende einer sich lebhaft bewegenden Larve eingetaucht, so bleibt dieselbe sofort wie gelähmt grade ausgestreckt liegen; lag sie schon vorher still, so erfolgt keine Veränderung. Herausnehmen der Cathode bewirkt vorübergehendes lebhaftes Schlängeln. - Wird die Cathode am Kopfende einer still liegenden oder sich bewegenden Larve plöt\%lich eingetaucht, so erfolgt lebhafte Unruhe, und meist Umkehrung der Larre, dann Ruhe. - Wird die Cathode zur Seite einer Larve eingetaucht, so erfolgen lebhafte Bewegungen, scheinbar ohne Gesetz; zuletzt legt sich meist die Larve so, dass sie der Cathode ihren Schwanz zuwendet.

Ist die bewegliche Electrode die Anode, so ist Alles entgegengesetzt: am Kopfende einer Larve versetzc sie dieselbe augenblicklich in lähmungsartige Ruhe; am Schwanzende versetzt sie die Larve in lebhafte Bewegung, die meist zur Umkehrung führt.

Sehr hübsch tritt die Verschiedenheit der Wirkung hervor, wenn, was in gut besetzten Trögen immer vorkommt, zwei Larven einander parallel, aber Kopf an Schwanz, dicht zusammen liegen, und man die eine Electrode plötzlich an das Kopfende der einen, also zugleich das Schwanzende der anderen bringt.

Eine andere vielfach von mir ausgeführte Form des Versuches ist die, dass man wiederum die bewegliche Electrode punct. förmig in der Nähe einzelner Larven eintaucht, aber den Strom erst nachher in der metallischen Leitung schliesst. Die Erscheinungen sind die gleichen, also z. B. macht die Anode am Kopf bei der Schliessung steifes Stilliegen der Larve, bei der Oeffnung Unruhe und Schlängeln. Bemerkenswerth ist, dass bei sehr 
starken Strömen zuweilen auch die Cathode, am Kopfe angebracht, steifes Stilliegen der Larve bewirkt.

Ebenso sieht man, wenn man beide Electroden vor oder nach der Schliessung punctförmig so eintaucht, dass eine Larve, oder ihr Rumpf allein, in der graden Verbindungslinie liegt, bei homodromer Lage, also absteigendem Strom, lebhafte Unruhe, bei antidromer Lage, also aufsteigendem Strom, steifes Stillliegen.

Werden beide Electroden zu beiden Seiten des Kopfes dicht an einer Larve eingetaucht, so sieht man, wie allch Herr Nenbauer unabhängig von mir bemerkte, die Larve sich etwas auf die Seite legen, und zwar die Anodenseite nach unten, die Cathodenseite nach oben; ich sah sogar einigemal einen Ansatz zu Rotationsbewegung in diesem Sinne. Diese Drehung ist offenbar gleichsinnig and innerlich verwandt mit der galvanischen Rotation böherer Thiere bei querer Durchströmung des Kopfes, und zeigt sich, wie Herr $\mathrm{N}$ e $\mathrm{u}$ bau e $\mathrm{r}$ constatirte, auch an ausgewachsenen Fröschen. Auf die Verwandtsehaft beider Erscheinungsreihen habe ich schon in der vorjährigen Mittheilung (S. 458) hingedeutet.

Ein Gesammt-Ueberblick der mitgetheilten Erscheinungen lehrt, dass sie sich auf das verschiedene Verhalten des Riickenmarks der Froschlarven gegen auf- und absteigende Ströme zurïckführen lassen. Der aufsteigende Strom bewirkt lebhafte Unruhe, welche sich zum Mindesten durch Schlängeln kundgiebt, der absteigende Strom wirkt dagegen nicht erregend, ja sogar wie es scheint bewegungshemmend oder lähmend. Vielleicht sind beide Wirkungen vorhanden, aber die letztere überwiegt. Herr Neubauer hat auch direct sehr feine Drähte an Kopf- und Schwanzende des Rumpfes angelegt, und bei aufsteigendem Strome Unruhe bis zum Tetanus, bei absteigendem nur Schliessungszuckung beobachtet. Nimmt man an, dass die Larven unter dem Einfluss der Ströme solche Lagen aufsuchen, bei welchen keine Erregung stattfindet, - ein Bestreben oder ein Instinct, welcher allen Geschöpfen eigen ist, - so muss es schliesslich dahin kommen, dass alle Larven sich so einstellen, dass sie absteigend durchflossen werden, d. h. dass sie den Kopf der Anode zuwenden. Die fundamentalere Erklärung des angefubrten Verhaltens des Rückenmarks gegen galvanische Ströme ziehe ich vor erst dann 
zu versuchen, wenn die bekanntlich noch sehr widerspruchsvollen entsprechenden Erscheinungen am Rückenmark erwachsener Frösche genügend festgestellt sind.

Bei dieser Gelegenheit sei es mir gestattet, einige andere Beobachtungen, welche ich an Froschlarven der ersten Wochen gemacht habe, kurz zu erwähnen.

Die Larven von Rana temporaria sind in dieser Zeit nngemein dunkel, fast schwarz. Einige Male aber fand ich sämmtliche im Troge befindliche Larven auffallend hell geworden, so dass sie fast durchsichtig waren, und das Herz deutlich roth und pulsirend von aussen gesehen werden konnte. Anfangs glaubte ich, diese Aufhellung sei eine Folge der vorausgegangenen galvanischen Reizversuche; allein da diese für gewöhnlich Nichts von solcher Wirkung zeigten, wurde ich auf eine andere Ursache aufmerksam. Die Versuche wurden nämlich zufällig im optischen Zimmer des Instituts angestellt, welches zuweilen zu anderen Versuchen vollständig verdunkelt wurde; es wurde constatirt, dass solche Verdunkelungen in den angegebenen Fällen unmittelbar vorausgegangen waren. Direct angestelle Versuche ergaben nun in der That, dass im Dunkeln die Larven regelmässig ganz hell und durchsichtig werden. Diese Wirkung erfolgt langsam, vollzieht sich aber in weniger als einer Stunde. Im Lichte werden sie etwas schneller wieder dunkel. Weiter fand ich, dass in rothem Lichte dies Dunkelwerden nicht eintritt, und dunkle Larven aus dem Tageslicht unter eine rothe Glasplatte gebracht, wie im Dunkeln hell werden. Blaues Licht wirkt dagegen wie Tageslicht. Indem ich hell gewordene Larven aus dem Dunkelraum direct im Sonnenlicht unter dem Microscop beobachtete, fand ich, dass die Pigmentkörper sich etwas in die Tiefe zurückgezogen hatten, und allmählich wieder der Oberfläche näher kamen. Zu weiterer Verfolgung dieser Erscheinung muss das nächste Frühahr abgewartet werden. Dieselbe erscheint deswegen bemerkenswerth, weil bei erwachsenen Fröschen die Verdunkelung meist als Folge von Lähmungserscheinungen beobachtet wird, während sie bei unsern Larven unter dem Einfluss des Lichtes, also wohl in Folge einer Erregung eintritt. Da abgeschnittene riickenmarklose Schwanzenden die Erscheinung nicht zeigten, scheint das Centralorgan bei derselben betheiligt zu sein. 
Die Froschlarven zeigen schon sehr frühzeitig (schon im Stadium der äusseren Kiemenbüschel) zwei deutliche Lungen, als längliche, am Ende zugespitzte, mit dem Schlunde communicirende Säckchen. So oft ich diese Organe präparirte, fand ich sie lufthaltig. Dies Verhalten ist auffallend, weil ich im Darmkanal niemals eine Spur von Luft fand. Wenn dieser Inhalt verschluckte Luft wäre (die Larven kommen allerdings häufig mit dem Manle an die Oberfläche des Wassers) oder von einem Luftgehalt der gefressenen Vegetabilien herrührte (die Larven sind äusserst gefrässig, ihr Darm ist stets mit grünnen Massen prall gefüllt, auch fressen sie begierig die Leichen ihrer Genossen), so müsste, sollte man meinen, auch der Darm lufthaltig sein. Vielleicht handelt es sich um eine Secretion von Gasen, nach Analogie der Schwimmblase.

Die Larven bilden ein gutes Object zum Studium der Flimmerbewegung, welche in der ersten Zeit an der ganzen Körperoberfläche vorhanden ist.

(Aus dem physiologischen Institut zu Königsberg i. Pr.)

\section{Ueber die Wirkung des Nitroprussidnatriums.}

$$
\text { Von }
$$

\section{Hermann.}

Vor $1 \frac{1 / 2}{2}$ Jahren habe ich die Beobachtung gemacht, dass kleine Dosen einer Lösung von Nitroprussidnatrium Warmbliiter unter den Erscheinungen der Blausäurevergiftung tödten, und dass die unmittelbar nach dem Tode geöffneten Höhlen des Thierkörpers einen intensiven Blausäuregeruch zeigen. Herr D avidsohn, welchem ich diesen Gegenstend zu weiterer Untersuchung ïbergeben habe, wird über denselben in seiner die Wirkung einiger Cyanverbindungen behandelnden Dissertation genauere Angaben machen. 